\title{
Revisión de la clasificación de Hinchey (diverticulitis) y su correlación terapéutica
}

\section{Hinchey's (Diverticulitis) Classification Review and it's Therapeutic Implacations}

\author{
Nicolás Daniel Roccatagliata ${ }^{1}$ [ Leidy Diana Rodríguez $^{1} \quad$ Lizeth Guardo $^{1} \quad$ Victor Nebil Larrañaga ${ }^{1}$ \\ Germán Espil ${ }^{1}$ Javier Vallejos ${ }^{1}$

\footnotetext{
${ }^{1}$ Centro de Educación Médica e Investigaciones Clínicas, Hospital Universitario Sede Saavedra, Departamento de Imágenes, Ciudad Autónoma de Buenos Aires, Argentina
} \\ Address for correspondence Nicolás Daniel Roccatagliata, MD, \\ Guatemala, 5731, Ciudad Autónoma de Buenos Aires, Argentina \\ (e-mail: nrocca@gmail.com).
}

Rev Argent Radiol 2020;84:123-129.

\section{Resumen}

Palabras clave

- diverticulitis

- colon

- tomografía computada

- tratamiento

\section{Abstract}

La diverticulosis es una entidad que predomina en países occidentales. Su prevalencia aumenta con la edad, presentándose en aproximadamente el $80 \%$ de la población mayor de 85 años. Los divertículos colónicos adquiridos son herniaciones saculares de la mucosa y submucosa (pseudodivertículos) y predominan en sigma, en países occidentales; los congénitos poseen las tres capas parietales (divertículos verdaderos) y predominan en colon derecho, en países asiáticos. Aproximadamente un 10\%-25\% de dichos pacientes con diverticulosis presentarán a lo largo de su vida un cuadro de diverticulitis aguda, representando una de las causas más frecuentes de abdomen agudo (3,8\%).

Clínicamente, se expresa por dolor abdominal en fosa ilíaca izquierda (excepción en dolicosigma/divertículos congénitos derechos) y pueden presentar complicaciones como flemones, abscesos, pileflebitis, peritonitis, con consiguiente riesgo de vida. La tomografía computada (TC) permite el diagnóstico oportuno, identificación de complicaciones y planificación terapéutica. A dicho fin se establecieron diferentes clasificaciones y modificaciones, siendo la más reconocida la propuesta por Hinchey (modificada por Wasvary y col., Kaiser y col.) y otras estableciendo correlaciones con el tratamiento, como la propuesta por Sartelli y col.

El objetivo del presente estudio es realizar una revisión iconográfica de esta última (Sartelli y col.) y evaluar sus implicancias terapéuticas.

Diverticular disease is an entity with high prevalence in western countries that increases with age, and affects approximately $80 \%$ of the population over 85 years of age. Acquired colonic diverticula are saccular mucosal and submucosal herniation (pseudodiverticles) and predominate in sigma, in western countries; the congenital ones possess the three parietal layers (true diverticula) and predominate in right side colon, in Asian countries. Approximately $10 \%-25 \%$ of patients with colonic diverticulosis, in their lifetime will present an episode of acute diverticulitis, which represents one of the most frequent causes of acute abdominal pain (3.8\%). received

August 24, 2019

accepted

April 15, 2020
DOI https://doi.org/

$10.1055 / \mathrm{s}-0040-1713089$.

ISSN 1852-9992.
Copyright @ $\odot$ 2020, Sociedad Argentina de Radiología. Publicado por Thieme Revinter Publicações Ltda., Rio de Janeiro, Brazil. Todos los derechos reservados.
License terms

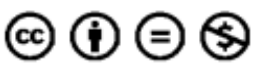


Clinically it express by abdominal pain in the left iliac fossa (exception in dolicosigma / right congenital diverticula) and may present complications such as phlegmon, abscesses, pylephlebitis, peritonitis, life threatening conditions.

\section{Keywords}

- colon

- diverticulitis

- computed tomography

- treatment
Computed tomography (CT) allows timely diagnosis, identification of complications and therapeutic planning. To this end, several classifications have been used, from which Hinchey's is the most renown (modified by Wasvary et al, Kaiser et al.), and other ones establish therapeutic correlation such as the one proposed by Sartelli et al.

The objective of the present study is to make an iconographic review of this last one (Sartelli et al.) and to evaluate its therapeutic implications.

\section{Discusión}

La diverticulosis es una entidad frecuente, que predomina en países occidentales, cuya prevalencia aumenta con la edad, presentándose en aproximadamente el 5\%-10\% de la población mayor a 45 años y el $80 \%$ de la población mayor a 85 años. ${ }^{1}$ Anatómicamente, se subdividen en divertículos verdaderos, los cuales presentan las tres capas parietales (mucosa, submucosa y muscular externa), hallazgo visualizado en los divertículos congénitos, que predominan en colon derecho, en población asiática, teniendo una prevalencia estable en los diferentes grupos etarios y representando aproximadamente un 5\%, y divertículos falsos, que se componen únicamente de mucosa y submucosa, como es el caso de la mayoría de los divertículos colónicos (aproximadamente 95\%), predominando en colon izquierdo y población occidental. ${ }^{2-4}$

Aproximadamente un 10\%-25\% de dichos pacientes con diverticulosis, a lo largo de su vida, presentarán un cuadro de diverticulitis aguda, representando una de las causas más frecuentes de abdomen agudo (3,8\%) y, por consiguiente, de gran impacto como problemática en salud pública. ${ }^{5}$

Clínicamente, dicho cuadro se caracteriza por dolor abdominal a predominio del cuadrante inferior izquierdo y fiebre, acompañado de alteración en los parámetros de laboratorio vinculables a un proceso infeccioso. En caso de complicaciones, puede progresar a un cuadro de peritonitis, presentando mal estado general, dolor a la descompresión, defensa abdominal. En ocasiones, se asocia además a hemorragia digestiva baja, estenosis post diverticulitis aguda, debiendo realizar el diagnóstico diferencial con patología neoplásica. ${ }^{6}$ Cuando la afectación compromete el colon derecho, clínicamente el cuadro es indistinguible de la apendicitis aguda, siendo los estudios por imágenes indispensables para un correcto diagnóstico ( - Fig. 1). ${ }^{7}$

A fin de establecer un lenguaje común entre los diferentes especialistas, en 1978 Hinchey y col. ${ }^{8}$ publicaron una clasificación basada principalmente en hallazgos clínicos y quirúrgicos (-Tabla 1).

Posteriormente, con el advenimiento y perfeccionamiento de nuevos métodos diagnósticos, se realizaron modificaciones a la clasificación original, en 1999 por Wasvary y col. ${ }^{9}$ y en 2005 por Kaiser y col. ${ }^{10}$, esta última de especial relevancia por la inclusión de hallazgos tomográficos (-Tabla 2). ${ }^{10}$ En la actualidad, la tomografía computada (TC) de abdomen y pelvis, sin y con contraste endovenoso es recomendada por la American College of Radiology en aquellos pacientes con

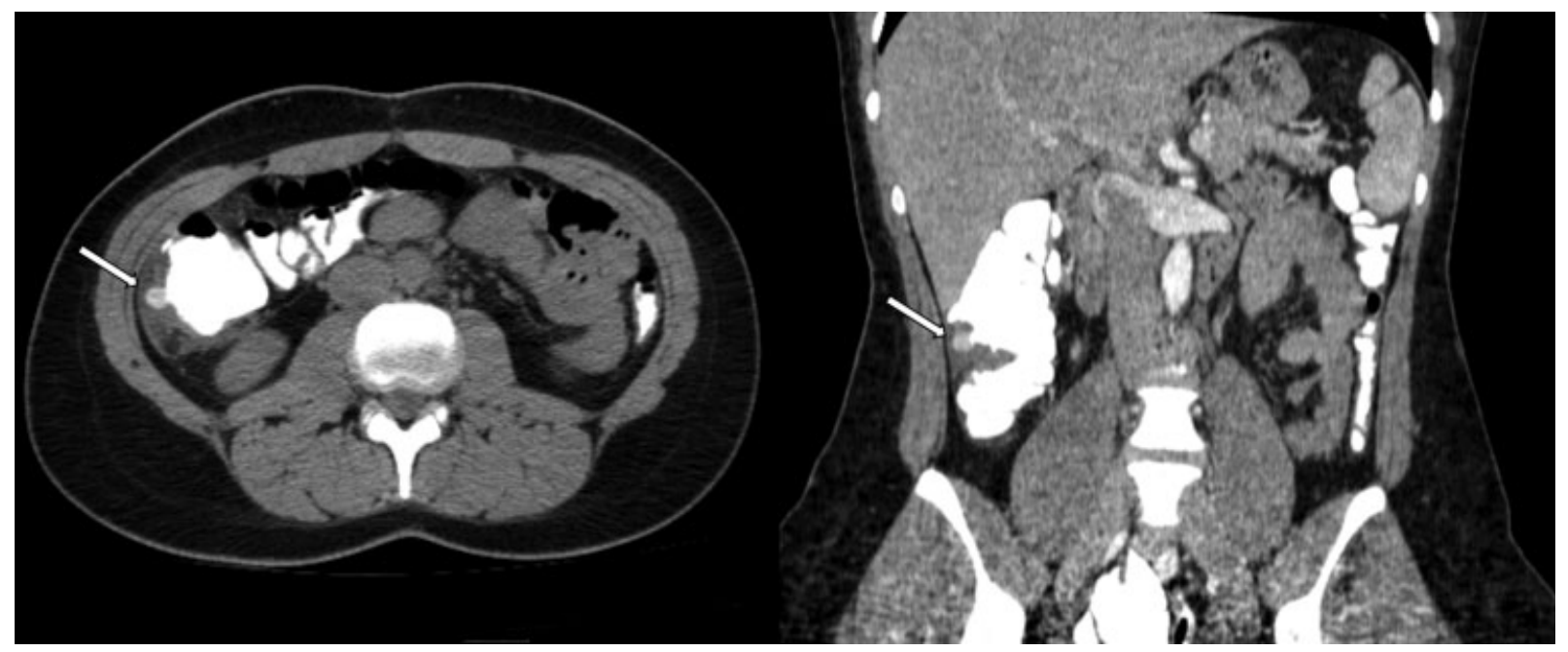

Fig. 1 Estadio IA. Paciente de 31 años se presenta al servicio de emergencias con cuadro de dolor abdominal agudo en flanco derecho y sospecha clínica de apendicitis. A nivel del colon ascendente, se visualiza imagen diverticular (flecha) asociada a engrosamiento parietal colónico, rarefacción de la grasa, imágenes ganglionares fuera de rango adenomegálico adyacentes y escasa cantidad de líquido libre laminar en gotiera parietocólica. 
Tabla 1 Clasificación de Hinchey y col. (1978)

\begin{tabular}{|l|l|}
\hline & Clasificación de Hinchey y col. $^{8}$ \\
\hline I & Absceso pericólico o flemón \\
\hline II & Absceso pélvico, abdominal o retroperitoneal \\
\hline III & Peritonitis generalizada purulenta \\
\hline IV & Peritonitis generalizada fecal \\
\hline
\end{tabular}

sospecha clínica, como estudio de imágenes inicial y para la correcta valoración de sus complicaciones. ${ }^{11}$

Con el objetivo de instaurar una correlación entre la clínica, los hallazgos tomográficos y el abordaje terapéutico, estableciendo el grado de severidad del cuadro con una visión multidisciplinaria, en 2015 Sartelli y col. ${ }^{12}$ propusieron una nueva clasificación y algoritmo de tratamiento ( - Fig. 2), la cual será expuesta a continuación. ${ }^{12}$

Tabla 2 Clasificación modificada por Wasvary y col. (1999), y por Kaiser y col. (2005)

\begin{tabular}{|l|l|l|}
\hline & Clasificación modificada por Wasvary y col. $^{9}$ & Clasificación modificada por Kaiser y col. (hallazgos tomográficos) $^{10}$ \\
\hline 0 & Engrosamiento de la pared colónica & Engrosamiento de la pared colónica \\
\hline Ia & Flegmón: inflamación pericólica confinada & Hallazgos de $0+$ cambios en el tejido pericólico \\
\hline Ib & Absceso pericólico o mesocólico & Hallazgos de la + Absceso pericólico o mesocólico \\
\hline II & $\begin{array}{l}\text { Absceso pélvico, abdominal } \\
\text { o retroperitoneal (absceso a distancia) }\end{array}$ & Hallazgos de la + Absceso a distancia \\
\hline III & Peritonitis generalizada purulenta & $\begin{array}{l}\text { Neumoperitoneo asociado a líquido libre localizado o ascitis } \\
\text { y posible engrosamiento parietal peritoneal }\end{array}$ \\
\hline IV & Peritonitis generalizada fecal & Hallazgos de III \\
\hline
\end{tabular}

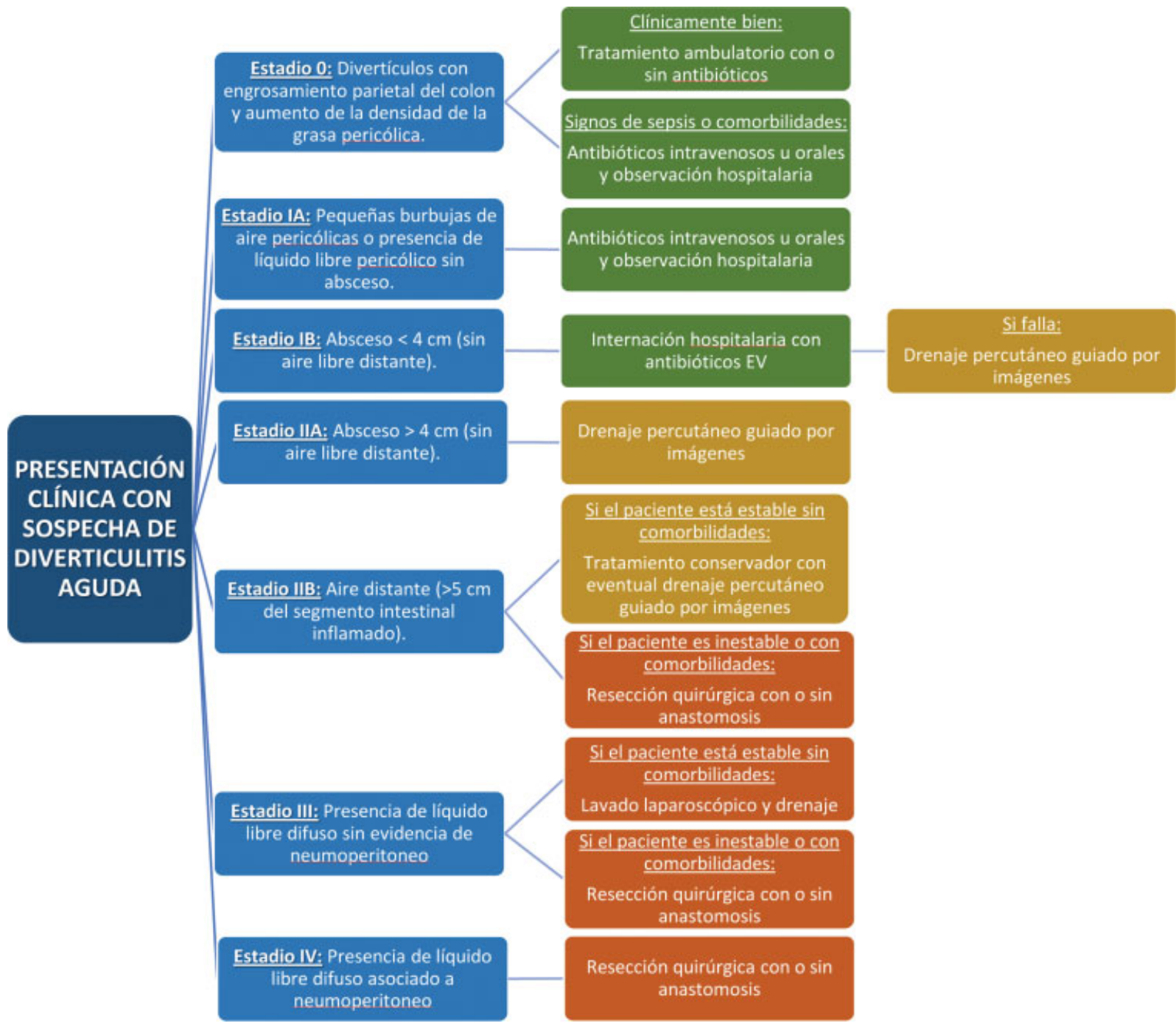

Fig. 2 Clasificación propuesta por Sartelli y col. (2015). 


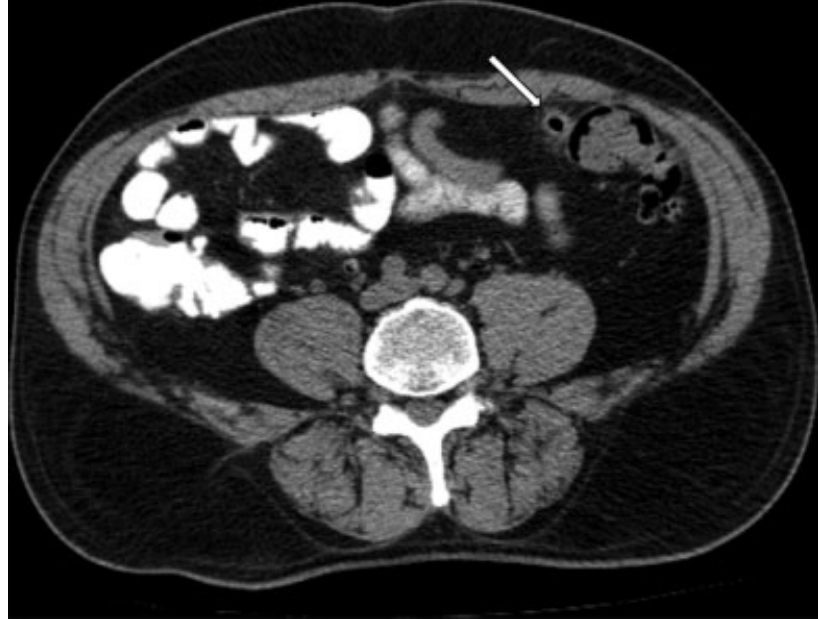

Fig. 3 Estadio 0. A nivel del colon sigmoide, en concordancia con región dolorosa referida por el paciente, se identifica imagen diverticular asociada a engrosamiento parietal (flecha), sin evidencia de cambios inflamatorios significativos loco-regionales.

- Estadio 0: Divertículos con engrosamiento parietal del colon y aumento de la densidad de la grasa pericólica. Si el paciente se encuentra en buen estado general, se recomienda el tratamiento ambulatorio con o sin antibióticos; en caso de presentar signos de sepsis o comorbilidades, hospitalización con administración de antibióticos por vía oral o intravenosa (-Fig. 3).

- Estadio IA: Pequeñas burbujas de aire pericólicas o presencia de líquido libre pericólico sin absceso. Se recomienda tratamiento con antibióticos intravenosos u orales y observación hospitalaria ( $\mathbf{- F i g . ~ 1 ) . ~}$

- Estadio IB: Absceso $<4 \mathrm{~cm}$ (sin aire libre distante). Se recomienda internación hospitalaria con antibióticos EV $\mathrm{y}$, en caso de fracaso terapéutico, drenaje percutáneo guiado por imágenes (ecografía o tomografía) (-Fig. 4).

- Estadio IIA: Absceso $>4 \mathrm{~cm}$ ( $\sin$ aire libre distante). Se recomienda drenaje percutáneo guiado por imágenes (-Figs. 5 y 6 ).
- Estadio IIB: Aire distante ( $>5 \mathrm{~cm}$ del segmento intestinal inflamado). Si el paciente está estable sin comorbilidades se recomienda tratamiento conservador con eventual drenaje percutáneo guiado por imágenes; en caso contrario, resección quirúrgica con o sin anastomosis (-Fig. 7).

- Estadio III: Presencia de líquido libre difuso sin evidencia de neumoperitoneo. Si el paciente está estable sin comorbilidades, se recomienda el lavado laparoscópico y drenaje; en caso contrario, resección quirúrgica con o sin anastomosis (-Fig. 8).

- Estadio IV: Presencia de líquido libre difuso asociado a neumoperitoneo. Se recomienda resección quirúrgica con o sin anastomosis (-Fig. 9).

Si bien el nuevo enfoque propuesto por Sartelli y col. ${ }^{12}$ resulta interesante a fin de establecer lenguaje común que permita un trabajo en conjunto entre los diferentes servicios y establecer una planificación terapéutica coordinada, en la actualidad la clasificación más utilizada y difundida continúa siendo la publicada por Kaiser y col. ${ }^{10}$

A pesar de ello, en los últimos años, se continuaron realizando estudios que correlacionan los hallazgos tomográficos con el tratamiento instaurado y el outcome obtenido. Un ejemplo de eso es el realizado por Lambrichts y col., donde se concluye que el tratamiento con drenaje percutáneo + antibiótico vs únicamente antibiótico no presentó diferencias significativas en pacientes con abscesos categorizados como Hinchey modificado Ib y II. ${ }^{14}$ También han surgido nuevas alternativas terapéuticas, como el abordaje endoscópico. ${ }^{2}$ Dichos estudios y la propuesta multidisciplinaria establecida por Sartelli y col. ${ }^{12}$ dejan abierto a discusión el enfoque clínico-radiológicoterapéutico a futuro de esa patología.

\section{Conclusión}

El conocimiento de la clasificación de Hinchey, sus modificaciones y nuevas clasificaciones propuestas (Sartelli

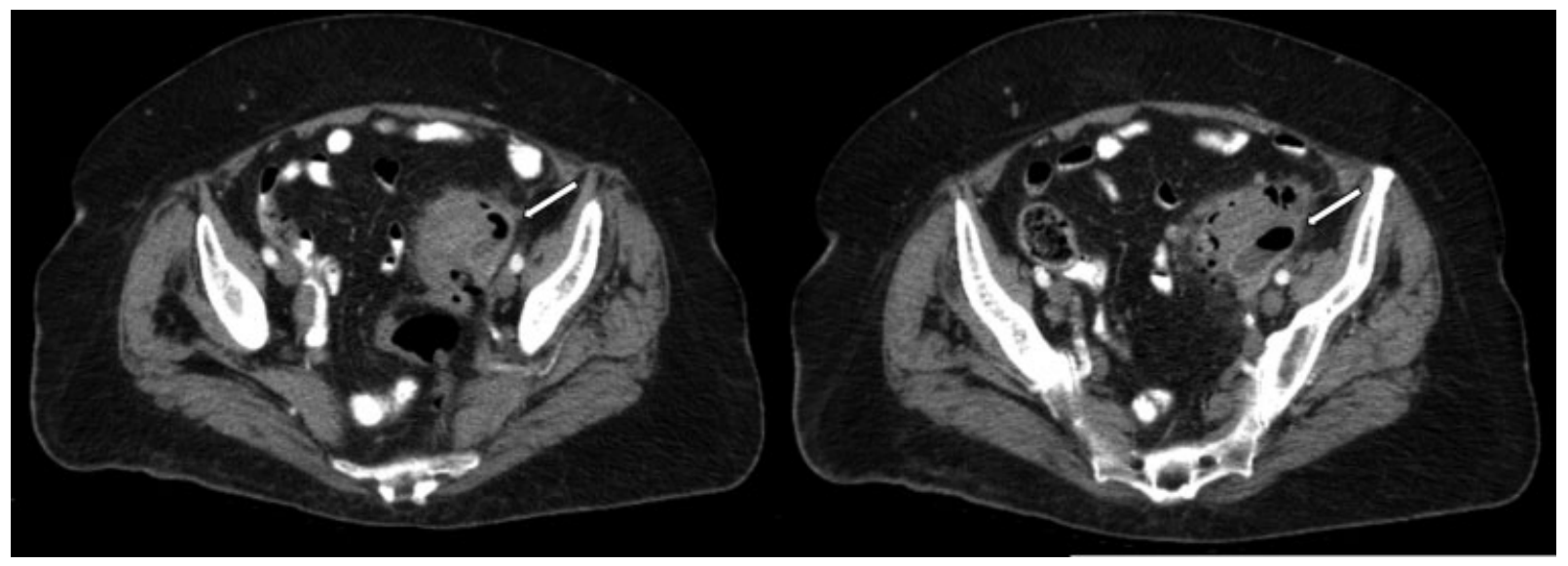

Fig. 4 Estadio IB. En colon sigmoideo se visualiza imagen diverticular y cambios inflamatorios loco-regionales (hallazgos de estadio IA) asociado a colección líquida (flecha) con aire y nivel hidroaéreo en su interior, la cual presenta realce periférico tras la administración de contraste endovenoso y mide aproximadamente $38 \times 16 \mathrm{~mm}$ en plano axial. 


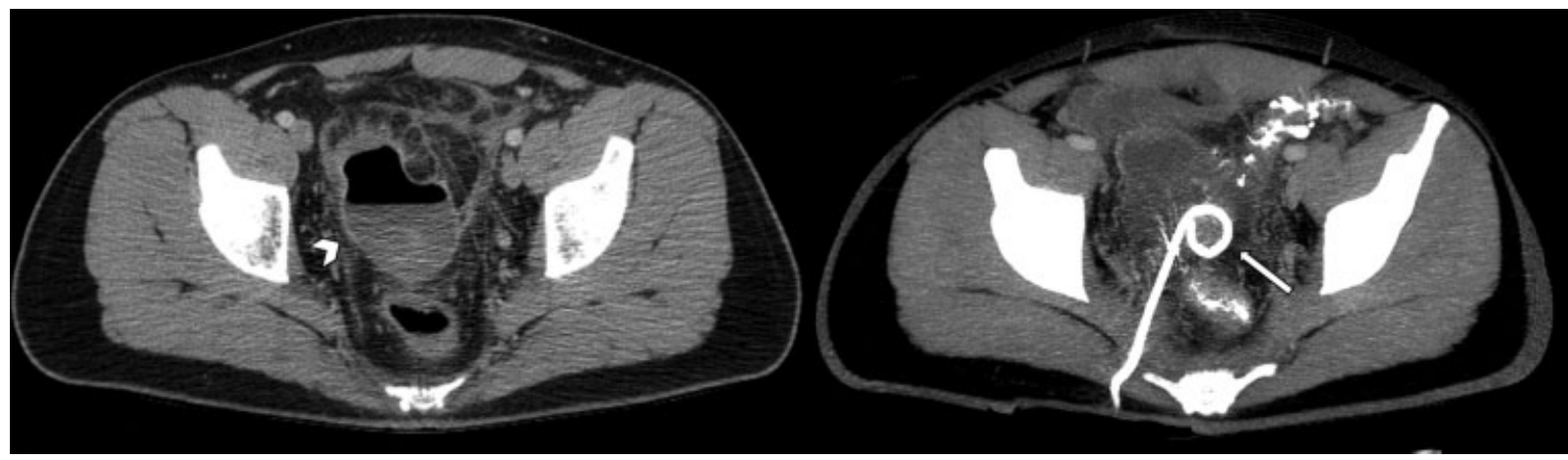

Fig. 5 Estadio IIA. En topografía del colon sigmoideo, se identifica imagen diverticular con cambios inflamatorios loco-regionales (hallazgos de estadio IA), destacándose a nivel de la pelvis, colección líquida (cabeza de flecha) con aire y nivel hidroaéreo en su interior, la cual presenta realce periférico tras la administración de contraste endovenoso y mide aproximadamente $76 \times 56 \mathrm{~mm}$ en plano axial. Se decide colocación percutánea de catéter Pig-Tail (flecha).

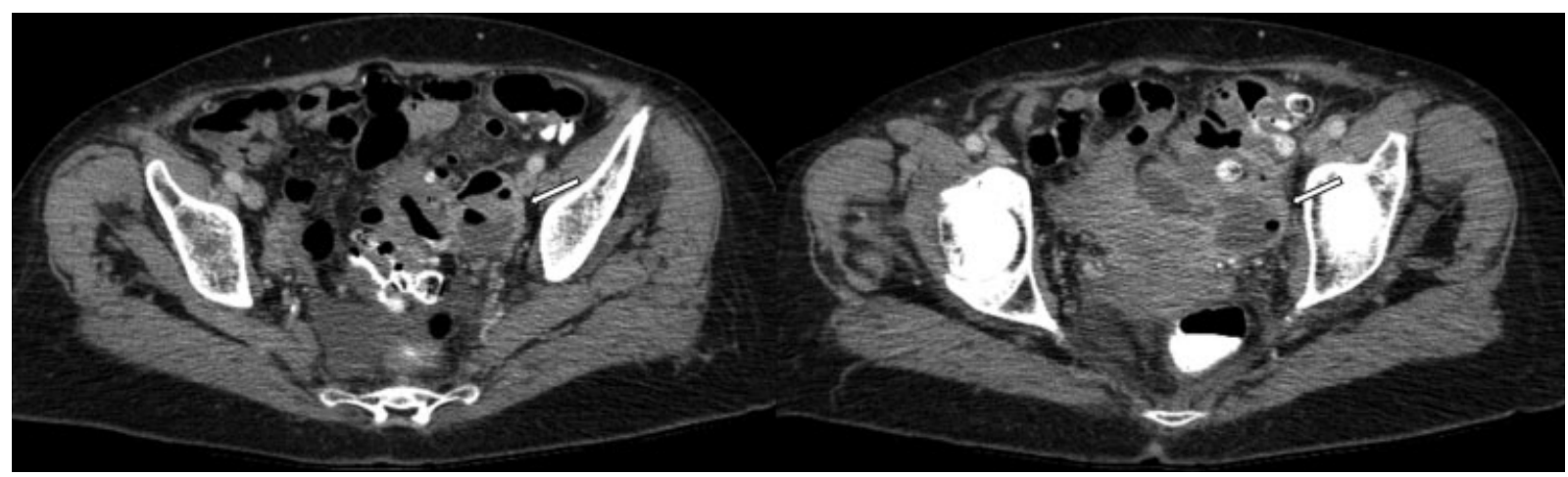

Fig. 6 Estadio IIA. En topografía del colon sigmoideo, se identifica imagen diverticular con cambios inflamatorios loco-regionales (hallazgos de estadio IA), visualizándose en área anexial izquierda, colección líquida (flecha) con niveles hidroaéreos, con realce periférico tras la administración de contraste endovenoso, la cual mide aproximadamente $43 \times 36 \mathrm{~mm}$ en plano axial.

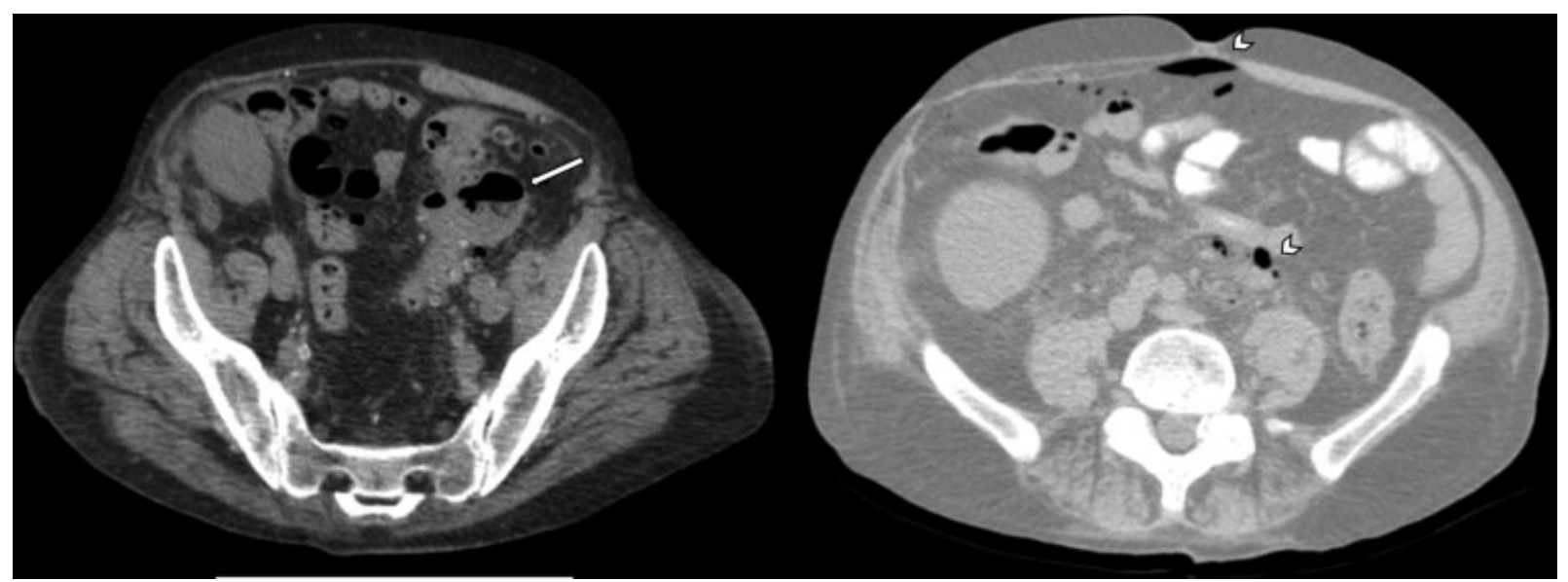

Fig. 7 Estadio IIB. Paciente trasplantado renal, presenta colección líquida pericólica (flecha) de $48 \times 32$ mm en plano axial (hallazgos de estadio IIA), asociándose a múltiples burbujas aéreas a distancia en cavidad peritoneal (cabeza de flecha).

y col. $^{12}$ ) permiten una adecuada evaluación de la diverticulitis aguda, sus complicaciones, hallazgos tomográficos y planificación terapéutica, estableciendo un nuevo enfoque multidisciplinario que permite un abordaje de la patología, coordinado y con un lenguaje común entre los diferentes servicios.

\section{Confidencialidad de los datos}

Los autores declaran que han seguido los protocolos de su centro de trabajo sobre la publicación de datos de pacientes y que todos los pacientes incluidos en el estudio han recibido información suficiente y han dado su consentimiento informado por escrito. 


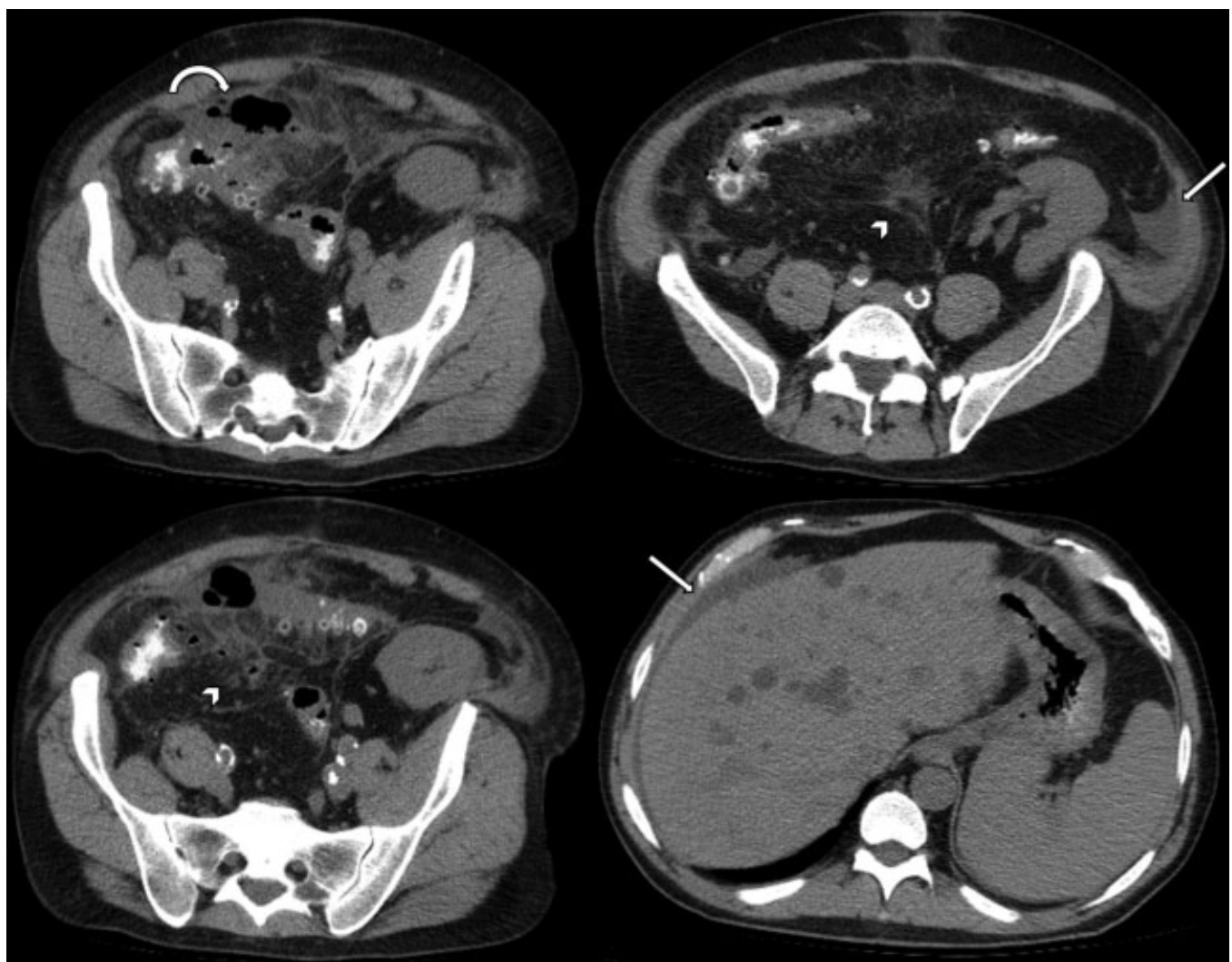

Fig. 8 Estadio III. Paciente trasplantado renal, presenta colección líquida pericólica (flecha curva) de $52 \times 29$ mm en plano axial (hallazgos de estadio IIA), asociado a rarefacción difusa de la grasa (cabeza de flecha) y líquido libre laminar loco-regional, perihepático y gotiera parietocólica izquierda (flechas rectas), sin evidencia de neumoperitoneo.

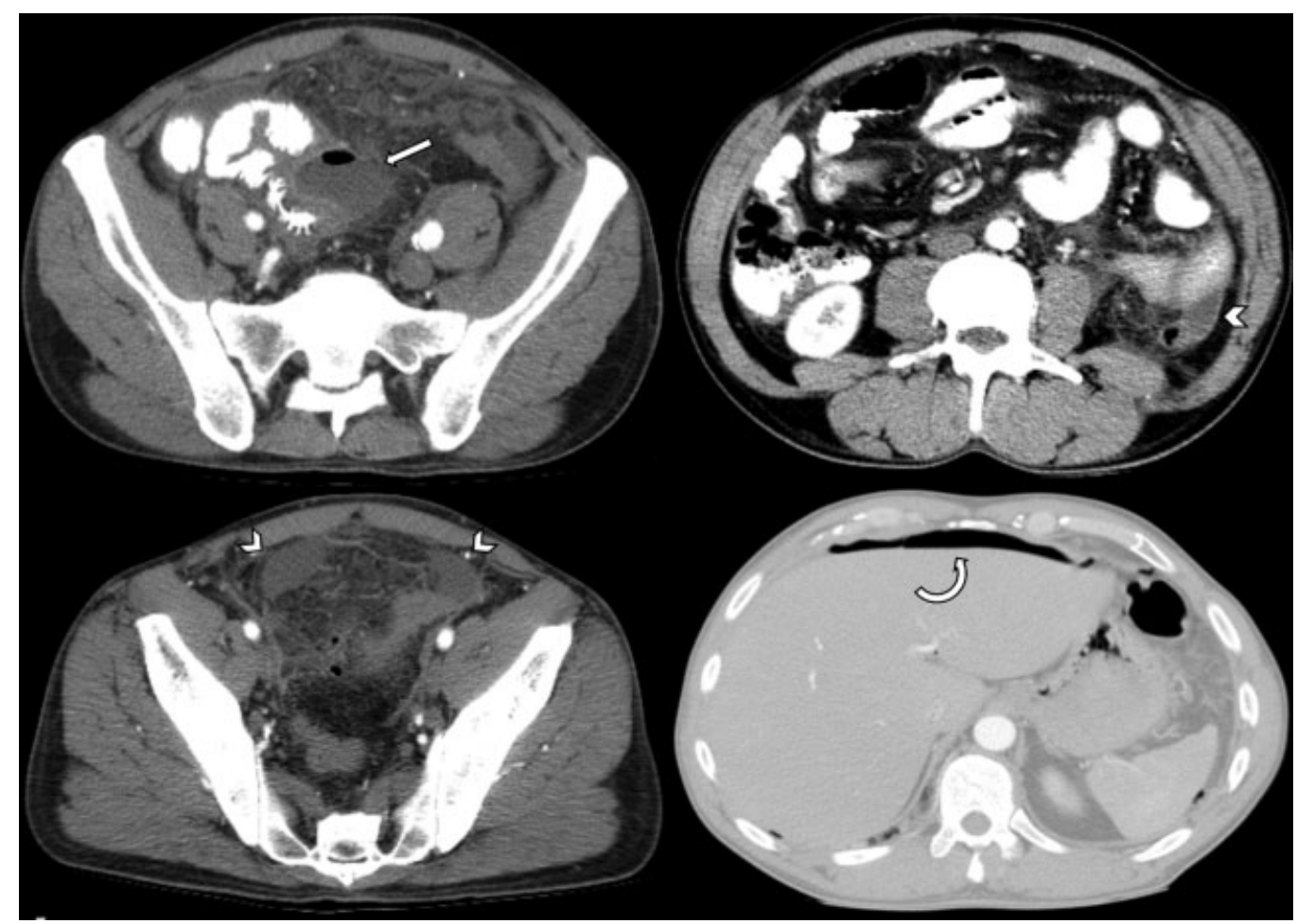

Fig. 9 Estadio IV. A nivel del colon transverso se identifica imagen diverticular con signos inflamatorios, asociada a colección pericólica (flecha recta) con nivel hidroaéreo, de $45 \times 27 \mathrm{~mm}$ en plano axial. Se identifica además rarefacción difusa de la grasa intraperitoneal, líquido libre perihepático, periesplénico, y en ambas gotieras parietocólicas con engrosamiento del peritoneo parietal (cabezas de flecha). Además, se observan signos de neumoperitoneo (flecha curva). 
Conflicto de Intereses

Los autores declaran no tener ningún conflicto de intereses.

\section{Bibliografía}

1 Horton KM, Corl FM, Fishman EK. CT evaluation of the colon: inflammatory disease. Radiographics. 2000;20(02):399-418

2 Zullo A, Gatta L, Vassallo R, Francesco V, Manta R, Monica F, et al. Paradigm shift: the Copernican revolution in diverticular disease. Ann Gastroenterol. 2019;32(06):541-553

3 Maykel JA, Opelka FG. Colonic diverticulosis and diverticular hemorrhage. Clin Colon Rectal Surg. 2004;17(03):195-204

4 Pereira JM, Sirlin CB, Pinto PS, Jeffrey RB, Stella DL, Casola G. Disproportionate fat stranding: a helpful CT sign in patients with acute abdominal pain. Radiographics. 2004;24(03):703-715

5 Onur MR, Akpinar E, Karaosmanoglu AD, Isayev C, Karcaaltincaba M. Diverticulitis: a comprehensive review with usual and unusual complications. Insights Imaging. 2017;8(01):19-27

6 Klarenbeek BR, de Korte N, van der Peet DL, Cuesta MA. Review of current classifications for diverticular disease and a translation into clinical practice. Int J Colorectal Dis. 2012;27(02):207-214

7 Chiu TC, Chou YH, Tiu CM, Chiou HJ, Wang HK, Lai YC, et al. Right-Sided Colonic Diverticulitis: Clinical Features, Sonographic
Appearances, and Management. J Med Ultrasound. 2017;25(01): 33-39

8 Hinchey EJ, Schaal PG, Richards GK. Treatment of perforated diverticular disease of the colon. Adv Surg. 1978;12:85-109

9 Wasvary H, Turfah F, Kadro O, Beauregard W. Same hospitalization resection for acute diverticulitis. Am Surg. 1999;65(07):632-635, discussion 636

10 Kaiser AM, Jiang JK, Lake JP, Ault G, Artinyan A, Gonzalez-Ruiz C, et al. The management of complicated diverticulitis and the role of computed tomography. Am J Gastroenterol.2005;100(04):910-917

11 Galgano SJ, McNamara MM, Peterson CM, Kim DH, Fowler KJ, Camacho MA, et al; Expert Panel on Gastrointestinal Imaging. ACR Appropriateness Criteria ${ }^{\circledR}$ Left Lower Quadrant Pain-Suspected Diverticulitis. J Am Coll Radiol. 2019;16(5S):S141-S149

12 Sartelli M, Moore FA, Ansaloni L, Di Saverio S, Coccolini F, Griffiths EA, et al. A proposal for a CT driven classification of left colon acute diverticulitis. World J Emerg Surg. 2015;10:3

13 Lambrichts DPV, Birindelli A, Tonini V, Cirocchi R, Cervellera M, Lange JF, et al. The Multidisciplinary Management of Acute Complicated Diverticulitis. Inflamm Intest Dis. 2018;3(02):80-90

14 Lambrichts DPV, Bolkenstein HE, van der Does DCHE, Dieleman D, Crolla RMPH, Dekker JWT, et al. Multicentre study of non-surgical management of diverticulitis with abscess formation. Br J Surg. 2019;106(04):458-466 